\title{
Treating symptoms to improve the quality of life in patients on chronic hemodialysis
}

\author{
Maurizio Bossola ${ }^{1}$ D $\cdot$ Gilda Pepe $^{2} \cdot$ Anna Picca $^{3} \cdot$ Riccardo Calvani $^{3} \cdot$ Emanuele Marzetti $^{3}$
}

Received: 18 December 2018 / Accepted: 4 March 2019 / Published online: 19 March 2019

(c) Springer Nature B.V. 2019

\begin{abstract}
Health-related quality of life (HRQOL) in patients on chronic hemodialysis has not improved significantly in the last 20 years. This is largely due to their substantial symptom burden which is rarely assessed and treated in routine clinical practice. This is also consequence of the lack of an appropriate armamentarium for the treatments of such symptoms. Adequate studies on the causes and pathogenesis of the symptoms of hemodialysis patients are needed followed by high-quality studies on possible therapeutic pharmacological and non-pharmacological interventions. Patients on chronic hemodialysis deserve a better quality of life.
\end{abstract}

Keywords Hemodialysis · Quality of life $\cdot$ Symptoms

In patients on chronic hemodialysis, health-related quality of life (HRQOL) is generally poor and significantly lower than that of the general population [1,2]. Unfortunately, HRQOL of these patients has not improved significantly in the last two decades [3-15]. Studies of large dialysis organizations found that HRQOL was essentially unchanged in the 2006 cohort compared to the 1996 one [3-5]. Accordingly, longitudinal studies have shown minimal variations of the HRQOL [6-15]. Specifically, the median changes in the physical (PCS) and mental (MCS) component summary scores were very low in the DOPPS study, and not clinically significant [13]. In the study of Liebman, mean score for PCS was unchanged, whereas MCS increased slightly [14]. In the HEMO study, there were no substantial variances in the PCS and MCS over 3 years [10]. Gabbay et al. found that self-reported HRQOL changed very little between 1997 and 2006, with no change in the SF-36 domains of general health, role physical, social functioning, and vitality, and

Maurizio Bossola

maurizio.bossola@gmail.com

1 Hemodialysis Unit, Institute of Clinical Surgery, Fondazione Policlinico Gemelli, Università Cattolica del Sacro Cuore, Largo A. Gemelli, 8, 00168 Rome, Italy

2 Department of Surgery, Fondazione Policlinico Gemelli, Università Cattolica del Sacro Cuore, Rome, Italy

3 Department of Gerontology, Fondazione Policlinico Gemelli, Università Cattolica del Sacro Cuore, Rome, Italy composite scores as PCS and MCS [1]. A clinically relevant change in HRQOL of five point or more (SF-36) for 2006 versus 1995 only for bodily pain, vitality, emotional, and mental health was demonstrated by Mazairac et al. [2].

Patients on chronic dialysis suffer from many physical and emotional symptoms [16-27]. Very few have described themselves as being symptom free while $35-50 \%$ of patients reported experiencing four to ten symptoms, and 25-35\% had more than ten [16]. The most common symptoms are fatigue, anorexia, pain, poor mobility, breathless, insomnia, cramps, anxiety, frustration, restless legs, and pruritus [16-27].

The high burden of physical and emotional symptoms in such patients is associated with a poor HRQOL [17-19] (Fig. 1). In one study, poor mobility and worse pain were the symptoms most closely associated with low HRQOL [17]. The major determinants of HRQOL, in other studies, were concurrent symptoms related to each other (symptom clusters) [20], the number [21] or the severity [18, 21, 24] of the symptoms.

Unfortunately, physicians are largely unaware of the presence of physical and emotional symptoms of patients on chronic hemodialysis or underestimate their severity [25-27]. In addition, symptoms are often undertreated in dialysis patients [25-27].

Discouragingly, the studies investigating the efficacy and safety of treatments of symptoms in patients on chronic hemodialysis are few. The number of studies, 


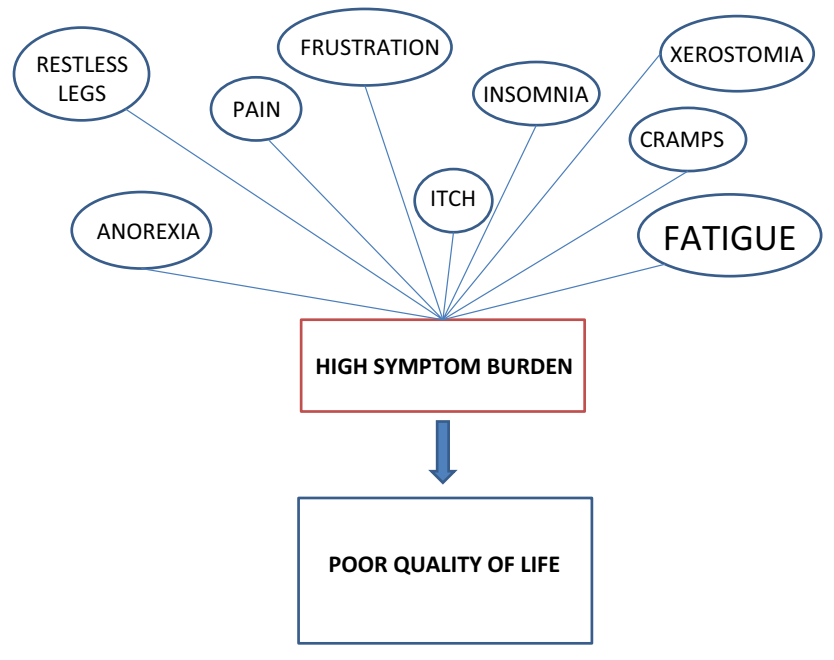

Fig. 1 Symptoms and quality of life in patients on chronic hemodialysis

performed between 1970 and 2018, on the treatment of each symptom as fatigue, itch, anorexia, xerostomia, restless legs, and insomnia is remarkably low. This is in net contrast with other chronic diseases. For instance, more than 200 studies have been published in the last 40 years about the treatment of fatigue in multiple sclerosis or more than 300 for cancer fatigue and even greater is the number of studies on the treatment of pain in patients with cancer or rheumatoid arthritis or with anorexia from cancer.

One reason for the paucity of such studies is the poor knowledge of the causes of the symptoms of hemodialysis patients. The underlying mechanisms of insomnia are unknown although it has been suggested that chronic inflammation, impaired altered metabolism of sleep-regulatory mediators, and sleep disruption related to treatment may be involved [28]. Increased systemic inflammation, abnormal serum parathyroid hormone, calcium, and phosphorus levels, an imbalance in opiate receptors, and a neuropathic process have been proposed contributing factors of uremic pruritus, its pathogenesis remains largely unclear [29]. With regard to fatigue, besides anemia and dialysis inadequacy, it has been suggested that it may be secondary to chronic inflammation and higher circulating levels of cytokines [30-32] but only one study has demonstrated such association [33]. Similarly, the mechanisms underlying uremia-related anorexia have been only in part elucidated and much remains to be clarified [34]. Xerostomia may be secondary to the use of some medications, but it seems to be due largely to a multifactorial mechanism, with the exact cause still remaining unknown [35]. Overall, it seems that when the causes and the mechanisms leading to the development of symptoms are essentially unknown, it is impossible to design and eventually test a therapeutic intervention.
In addition, when present, the studies on the treatment of symptoms of patients on chronic hemodialysis, such as fatigue, insomnia and sleep disorders, xerostomia, and restless legs often lead to the same conclusion that is lack of results and indication for therapy. On insomnia, Lindner et al. have concluded that "limited intervention trials are available to establish an appropriate evidence base for specific treatment recommendations" [28]. "To date, data on interventions on renal patients are sparse and definitive conclusions cannot be drawn due to the limited number of studies and their lack of generalizability to the wider population" is the disappointing final statement of the Artom et al. on fatigue [32]. The authors' conclusions of the review on antidepressant for treating depression in patients on chronic hemodialysis are that "despite the high prevalence of depression in dialysis patients, evidence for antidepressant medication in the dialysis setting is sparse and data are generally inconclusive. The relative benefits and harms of antidepressant therapy are poorly known and large randomized studies of antidepressant versus placebo are required" [36]. With regard to RLS, Sakkas et al. recently concluded that only exercise has been proven beneficial in both reducing the RLS symptoms' severity score and improving the quality of life [37]. Bossola et al. concluded, in their review about xerostomia, that the results from the few studies conducted on treatments of this frequent and debilitating symptom have been contradictory or inconclusive [35].

In conclusion, effort should be made to design adequate studies on the causes and pathogenesis of physical and emotional symptoms of patients on chronic hemodialysis. Once the underlying mechanisms are identified, it is mandatory to perform high-quality studies on possible therapeutic pharmacological and non-pharmacological interventions. In this program, associations of patients should have a strong and stimulating role. In this regard, a model that might be followed is what has been done by patients' association in HIV infection. Patients on chronic dialysis deserve urgent and adequate answers to their need for a better quality of life.

\section{Compliance with ethical standards}

Conflict of interest All authors declare no conflict of interest.

Research involving in human and animal participants This article does contain any studies with human performed by any of the authors.

\section{References}

1. Gabbay E, Meyer KB, Griffith JL, Richardson MM, Miskulin DC (2010) Temporal trends in health-related quality of life among 
hemodialysis patients in the United States. Clin J Am Soc Nephrol 5:261-267

2. Mazairac AH, de Wit GA, Penne EL et al (2011) Changes in quality of life overtime-Dutch haemodialysis patients and general population compared. Nephrol Dial Transplant 26:1984-1989

3. Lacson E Jr, Xu J, Lin SF, Dean SG, Lazarus JM, Hakim R (2009) Association between achievement of hemodialysis qualityof-care indicators and quality-of-life scores. Am J Kidney Dis 54:1098-1107

4. Diaz-Buxo JA, Lowrie EG, Lew NL, Zhang H, Lazarus JM (2000) Quality-of-life evaluation using short form 36: comparison in hemodialysis and peritoneal dialysis patients. Am J Kidney Dis 35:293-300

5. Lowrie EG, Curtin RB, LePain N, Schatell D (2003) Medical outcomes study short form-36: a consistent and powerful predictor of morbidity and mortality in dialysis patients. Am J Kidney Dis 41:1286-1292

6. Merkus MP, Jager KJ, Dekker FW, de Haan RJ, Boeschoten EW, Krediet RT (1999) Physical symptoms and quality of life in patients on chronic dialysis: results of the netherlands cooperative study on adequacy of dialysis (NECOSAD). Nephrol Dial Transplant 14:1163-1170

7. Bakewell AB, Higgins RM, Edmunds ME (2002) Quality of life in peritoneal dialysis patients: decline over time and association with clinical outcomes. Kidney Int 61:239-248

8. Wu AW, Fink NE, Marsh-Manzi JV et al (2004) Changes in quality of life during hemodialysis and peritoneal dialysis treatment: generic and disease specific measures. J Am Soc Nephrol 15:743-753

9. Garcia-Mendoza M, Valdés C, Ortega T, Rebollo P, Ortega F (2006) Differences in health-related quality of life between elderly and younger patients on hemodialysis. J Nephrol 19:808-818

10. Unruh ML, Newman AB, Larive B et al (2008) The influence of age on changes in health-related quality of life over three years in a cohort undergoing hemodialysis. J Am Geriatr Soc 56:1608-1617

11. Santos PR, Daher EF, Silva GB Jr, Libório AB, Kerr LR (2009) Quality of life assessment among haemodialysis patients in a single centre: a 2-year follow-up. Qual Life Res 18:541-546

12. Broers NJ, Usvyat LA, Kooman JP et al (2015) Quality of life in dialysis patients: a retrospective cohort study. Nephron 130:105-112

13. Perl J, Karaboyas A, Morgenstern H et al (2017) Association between changes in quality of life and mortality in hemodialysis patients: results from the DOPPS. Nephrol Dial Transplant 32:521-527

14. Liebman S, Li NC, Lacson E (2016) Change in quality of life and one-year mortality risk in maintenance dialysis patients. Qual Life Res 25:2295-2306

15. Belayev LY, Mor MK, Sevick MA et al (2015) Longitudinal associations of depressive symptoms and pain with quality of life in patients receiving chronic hemodialysis. Hemodial Int 19:216-224

16. Murtagh FE, Addington-Hall J, Higginson IJ (2007) The prevalence of symptoms in end-stage renal disease: a systematic review. Adv Chronic Kidney Dis 14:82-99

17. Lowney AC, Myles HT, Bristowe K et al (2015) Understanding what influences the health-related quality of life of hemodialysis patients: a collaborative study in England and Ireland. J Pain Symptom Manag 50:778-785

18. Weisbord SD, Fried LF, Arnold RM et al (2005) Prevalence, severity, and importance of physical and emotional symptoms in chronic hemodialysis patients. J Am Soc Nephrol 16:2487-2494
19. Abdel-Kader K, Unruh ML, Weisbord SD (2009) Symptom burden, depression, and quality of life in chronic and end-stage kidney disease. Clin J Am Soc Nephrol 4:1057-1064

20. Thong MS, van Dijk S, Noordzij M et al (2009) Symptom clusters in incident dialysis patients: associations with clinical variables and quality of life. Nephrol Dial Transplant 24:225-230

21. Yong DS, Kwok AO, Wong DM, Suen MH, Chen WT, Tse DM (2009) Symptom burden and quality of life in end-stage renal disease: a study of 179 patients on dialysis and palliative care. Palliat Med 23:111-119

22. Davison SN, Jhangri GS, Johnson JA (2006) Cross-sectional validity of a modified edmonton symptom assessment system in dialysis patients: a simple assessment of symptom burden. Kidney Int 69:1621-1625

23. Weisbord SD, Fried LF, Unruh ML et al (2007) Associations of race with depression and symptoms in patients on maintenance haemodialysis. Nephrol Dial Transplant 22:203-208

24. Weisbord SD, Bossola M, Fried LF et al (2008) Cultural comparison of symptoms in patients on maintenance hemodialysis. Hemodial Int 12:434-440

25. Claxton RN, Blackhall L, Weisbord SD, Holley JL (2010) Undertreatment of symptoms inpatients on maintenance hemodialysis. J Pain Symptom Manag 39:211-218

26. Weisbord SD, Fried LF, Mor MK et al (2007) Renal provider recognition of symptoms in patients on maintenance hemodialysis. Clin J Am Soc Nephrol 2:960-967

27. Raj R, Ahuja KD, Frandsen M, Jose M (2017) Symptoms and their recognition in adult haemodialysis patients: interactions with quality of life. Nephrology (Carlton) 22:228-233

28. Lindner AV, Novak M, Bohra M, Mucsi I (2015) Insomnia in patients with chronic kidney disease. Semin Nephrol 35:359-372

29. Combs SA, Teixeira JP, Germain MJ (2015) Pruritus in kidney disease. Semin Nephrol 35:383-391

30. Bossola M, Tazza L. (2016) Postdialysis fatigue: a frequent and debilitating symptom. Semin Dial 29(3):222-227

31. Bossola M, Vulpio C, Tazza L (2011) Fatigue in chronic dialysis patients. Semin Dial 24(5):550-555

32. Artom M, Moss-Morris R, Caskey F, Chilcot J (2014) Fatigue in advanced kidney disease. Kidney Int 86:497-505

33. Bossola M, Di Stasio E, Giungi S, Rosa F, Tazza L (2015) Fatigue is associated with serum interleukin-6 levels and symptoms of depression in patients on chronic hemodialysis. J Pain Symptom Manag 49:578-585

34. Bossola M, Giungi S, Luciani G, Tazza L (2011) Interventions to counteract anorexia in dialysis patients. J Ren Nutr 21:16-19

35. Bossola M, Tazza L (2012) Xerostomia in patients on chronic hemodialysis. Nat Rev Nephrol 8:176-182

36. Palmer SC, Natale P, Ruospo M et al (2016) Antidepressants for treating depression in adults with end-stage kidney disease treated with dialysis. Cochrane Database Syst Rev 23:CD004541

37. Sakkas GK, Giannaki CD, Karatzaferi C, Maridaki M, Koutedakis Y, Hadjigeorgiou GM, Stefanidis I (2015) Current trends in the management of uremic restless legs syndrome: a systematic review on aspects related to quality of life, cardiovascular mortality and survival. Sleep Med Rev 21:39-49

Publisher's Note Springer Nature remains neutral with regard to jurisdictional claims in published maps and institutional affiliations. 